\title{
Human Visual Perception of Two-Dimensional Symmetry
}

\author{
Jeremy Cole (jrcole@psu.edu) and David Reitter (reitter@psu.edu) \\ College of Information Sciences and Technology
}

Yanxi Liu (yanxi@ cse.psu.edu)

Department of Computer Science and Engineering

The Pennsylvania State University, University Park, PA 16802, USA

\begin{abstract}
Most literature on symmetry perception has focused on bilateral reflection symmetry with some suggesting that it is the only type of symmetry humans can perceive (Wilson \& Wilkinson, 2002). Using image stimuli generated from the mathematically well-defined seventeen wallpaper groups, this study demonstrates that humans can discriminate various symmetries found in 2D wallpaper patterns (Liu, Hel-Or, Kaplan, Van Gool, et al., 2010). Furthermore, the results demonstrate the features which contribute to wallpaper pattern perception. All wallpaper groups but one were found to be reliably distinguishable $(p<0.05)$. Additionally, as wallpaper patterns can be arranged in a hierarchy, we propose a metric to quantify the similarity of their perception using the shortest path in this hierarchy. This subgroup distance was found to be a factor in a likely model of pattern perception.
\end{abstract}

Keywords: symmetry; visual perception; computational cognition

\section{Introduction}

The concept of symmetry refers to a common property of everyday objects and images. Symmetry is present when, for instance, one half of the object appears mirrored along an axis. Faces are a good example: they are approximately symmetrical along a vertical axis. Symmetry has received much attention as a feature of visual perception and attention (see (Treder, 2010) for a review). However, symmetry as a geometric concept is much more general: symmetry refers to any transformation of an object or image that leaves it with the exact same appearance. Mathematically, exactly four types of symmetry are distinguished in two dimensional images.

Prior work has focused largely on reflection. It has even been suggested that this is the only type of symmetry humans can immediately process (Wilson \& Wilkinson, 2002). In this study, we present empirical data suggesting that this is not the case. The experiments are designed to test whether people perceive differences in symmetry, and whether they can identify and distinguish all groups of objects that are formed by two dimensional symmetries.

The common term symmetry generally refers to reflection, with many studies focused on bilateral reflection. Bilateral reflection describes objects where a single vertical axis separates two mirrored halves. Reflection is one of the four types of two dimensional symmetry; the others are rotation, glide reflection, and translation. As symmetry is a transformation that leaves the object the same, this can apply to objects beyond even images. For instance, if we consider an infinitely long loop of musical notes, shifting all the notes forward in time by the length of the loop would not change the music at all. However, this paper focuses on symmetry as it relates to two dimensional images.

We will use wallpapers to create images that use combinations of these symmetry types. Wallpapers are twodimensional images made from an infinitely repeating symmetric tile. These tiles are characterized by a specific set of symmetries. There are exactly seventeen wallpaper groups, as has been well-known for over a hundred years (Federov, 1891). In other words, every two-dimensional repeating image has one of seventeen sets of symmetries. All wallpapers can be arranged in a hierarchy, as shown in Figure 1 (Liu et al., 2010).

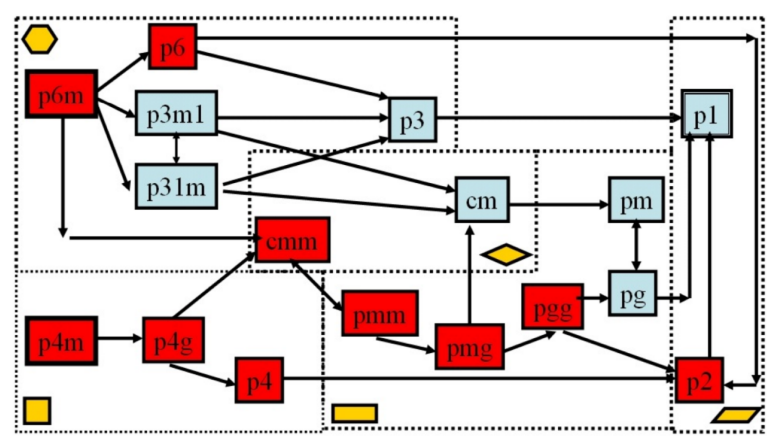

Figure 1: A hierarchy of all of the supergroup-subgroup relationships. An arrow means that the origin group's symmetries is a superset of the destination group's symmetries.

Through statistical modeling of our empirical data, we will argue that this mathematically well-motivated hierarchy of symmetry groups plays a role in perceptual processing.

\section{Prior Work}

Despite the age of the mathematical work on the topic, little has been done to connect these patterns to human perception. Symmetry beyond bilateral reflection has received little attention. This is perhaps due to work that suggests faces played a special role in our brain's evolution (Kanwisher, McDermott, \& Chun, 1997); bilateral reflection is the only symmetry they contain. There are several works covering bilateral reflection symmetry. Ferguson, Aminoff, and Gentner (1996) explain that qualitative features are more important than quantitative differences in symmetry judgments. Morales and Pashler (1999) discusses that having to check color increases the 


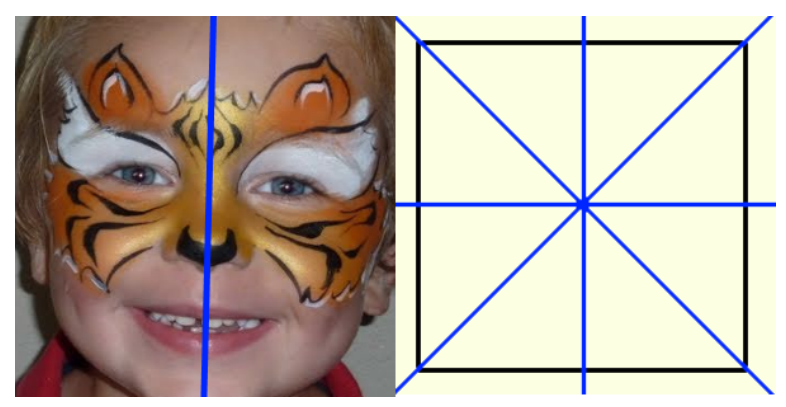

Figure 2: On the left, a face, exhibiting bilateral vertical reflection symmetry. On the right, a square, which contains four reflection axes. Reflection axes are marked with blue lines. Notice that mirroring the images about any of the axes would result in the same image

time it takes someone to determine whether an image is symmetric. They used this to argue that humans do not use color when making such judgments.

However, Wilson and Wilkinson (2002) criticized these and other studies for relying on shapes that are not biological, which they claim is the origin of symmetry detection. Generally, many studies use blocks or other generated images rather than those of naturally occurring objects, such as faces or flowers. The authors further make the argument that bilateral vertical reflection is the only symmetry that has much prevalence in nature. While the examples provided should give some evidence of other types of symmetry in nature, we also seek to counter the claim that humans are not good at detecting other types of symmetry.

The only study that we know of specifically dealing with wallpaper groups is Clarke, Green, Halley, and Chantler (2011). In that study, Clarke et al. found that subjects did not perform well in a symmetry group sorting task. Further, he found that they mostly used rotation in distinguishing among the groups. In this paper, we argue that the nature of Clarke's task was difficult for reasons other than perceiving the difference between two groups. Further, we argue that the strategy is not simply based on rotation.

\section{Background}

In two-dimensional images, there are only four symmetric transformations that are not a composition of other transformations. These are reflection, rotation, glide reflection, and translation. Various combinations of these symmetries are what differentiate the seventeen wallpaper groups. We note that human perception does not necessarily rely on perfect symmetry. While textures which have been manipulated can pose a serious problem for computer vision algorithms, humans are quite good at recognizing patterns even in significantly distorted images (Liu, Lin, \& Hays, 2004).

Reflection is the most well-known symmetry, with the vernacular for symmetry referring to it exclusively. Objects that contain reflection have a reflection axis, a line that separates the image into two mirrored halves. Examples of reflection
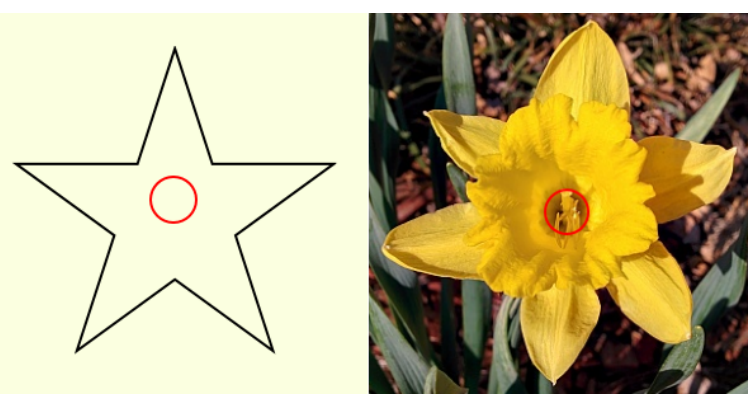

Figure 3: On the left, a flower exhibiting 6-fold rotation symmetry. On the right, a six-pointed star, exhibiting 6-fold rotation symmetry. Rotation centers for each are marked with red circles

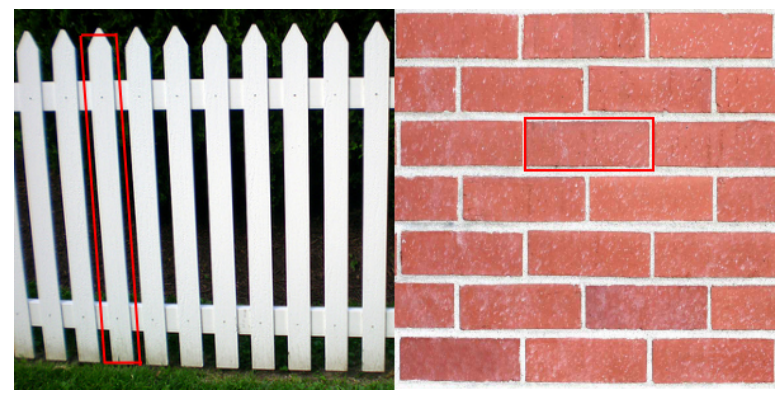

Figure 4: On the left, a white fence. On the right, a brick wall. In both of these images, the image would have to repeat infinitely to truly exhibit translation symmetry. The tile is outlined in red. In the brick wall, the brick could be shifted along two separate axes, while in the white fence, it would have to be shifted left or right.

symmetry in nature include the faces and bodies of animals. Reflection is often characterized by the number of reflection axes that can divide the object. A face, for instance, would only contain one. A square, on the other hand, would contain three (see Figure 2).

Rotation symmetry also frequently in nature. Rotation symmetry refers to an object's ability to rotate around some center without changing. It is characterized by the number of rotation angles that maintain this symmetry. For instance, a hexagon and a flower with six pedals would both have 6-fold rotation symmetry (see Figure 3). On the other hand,

Translation symmetry is when an object contains repeating tiles. Tiles are small identical pieces of the image that repeat. For instance, in a brick wall, a single brick could be the tile. An infinitely long brick wall contains symmetry because shifting every brick to the right by the length of one brick leaves the wall appearing exactly the same. Another example of translation symmetry is a fence. If the fence was shifted by the length from one post to the next, the fence would stay the same. See Figure 4 for examples.

The last basic symmetry is glide reflection. Glide reflection requires translation symmetry to exist. While reflection refers to an object directly mirrored across an axis, glide re- 


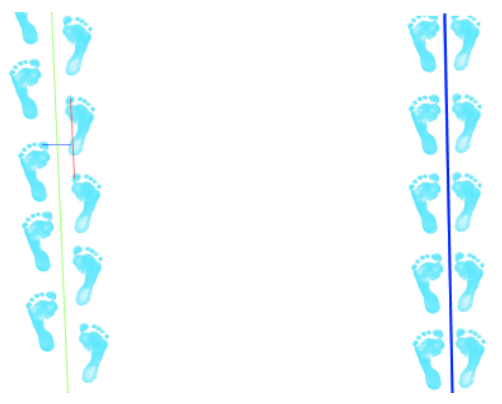

Figure 5: On the left, footsteps exhibiting a glide reflection pattern. On the right, footsteps exhibiting a normal reflection pattern. Note that along any given axis, only one is possible. If its mirrored after translation, it is glide reflection. If it is mirrored without translation, it is normal reflection.

flection is when the object is mirrored at exactly half of the translation distance. One example of glide reflection in nature are footsteps. Halfway in between each footstep, there is a reflected footstep (made by the other foot). Importantly, it is impossible to have glide reflection and reflection along the same axis. For instance, if two sets of footsteps form exact mirrors of each other, then that axis is said to have reflection, not glide reflection (see Figure 5).

In the wallpaper groups, every group has translation symmetry and a unique set of other symmetries. The seventeen groups comprise all possible sets. Table 1 gives a description of the seventeen groups in terms of their symmetries. Notably, sometimes even when both groups have reflection, they have it along different axes. See Figure ?? for an example. Importantly, any single group would have the exact same symmetries, even if their appearance was different. See Figure ?? for an example. Every group also has its own tile shape, which is the shape of the smallest repeating piece of the image.

In representing wallpaper groups as a set of symmetries, we can form relationships among the groups based on these sets. Every group's symmetries are either a subset or a superset of every other group. If each group is placed with its relation to other groups, they form a graph, see Figure 6. Note that replacing glide reflection with reflection along an axis maintains the superset relationship. See Table 1 to otherwise see that this hierarchy is formed. Note that the tile shape is not part of the group-theoretic analysis.

\section{Research Questions}

Due to previous work not focusing on the role of symmetry groups in perception, we sought to design an experiment that tested which types of symmetry are easily perceived by humans. We also wanted to compare multiple theories of symmetry perception. In order to do this, we designed a task requiring people to differentiate wallpaper groups. Our research questions can be summarized as:

\begin{tabular}{|l|c|c|c|c|c|c|c|c|c|}
\hline Group & 2-fold & 3-fold & 4-fold & 6-fold & $T_{1}$ & $T_{2}$ & $D_{1}$ & $D_{2}$ & tile \\
\hline P1 & F & F & F & F & None & None & None & None & O \\
\hline P2 & T & F & F & F & None & None & None & None & O \\
\hline PM & F & F & F & F & Refl & None & None & None & Re \\
\hline PG & F & F & F & F & Glide & None & None & None & Re \\
\hline CM & F & F & F & F & None & None & Refl & None & Rh \\
\hline PMM & T & F & F & F & Glide & Refl & None & None & Re \\
\hline PMG & T & F & F & F & Glide & Refl & None & None & Re \\
\hline PGG & T & F & F & F & Glide & Glide & None & None & Re \\
\hline CMM & T & F & F & F & None & None & Refl & Refl & Rh \\
\hline P4 & T & F & T & F & None & None & None & None & S \\
\hline P4M & T & F & T & F & Refl & Refl & Refl & Refl & S \\
\hline P4G & T & F & T & F & Glide & Glide & Refl & Refl & S \\
\hline P3 & F & T & F & F & None & None & None & None & H \\
\hline P3M1 & F & T & F & F & None & None & Refl & None & H \\
\hline P31M & F & T & F & F & Refl & Refl & Refl & None & H \\
\hline P6 & T & T & F & T & Refl & None & None & None & H \\
\hline P6M & T & T & F & T & Refl & Refl & Refl & Refl & H \\
\hline
\end{tabular}

Table 1: Wallpaper groups represented as their symmetries. The first four columns are whether the group has that type of rotation symmetry. The second four columns refer to the four main axes on the tile. (Refl=Reflection, Glide=Glide Reflection, $\mathrm{Re}=$ Rectangular, $\mathrm{Rh}=$ Rhombic, $\mathrm{O}=$ Oblique, $\mathrm{S}=$ Square, $\mathrm{H}=$ Hexagonal)

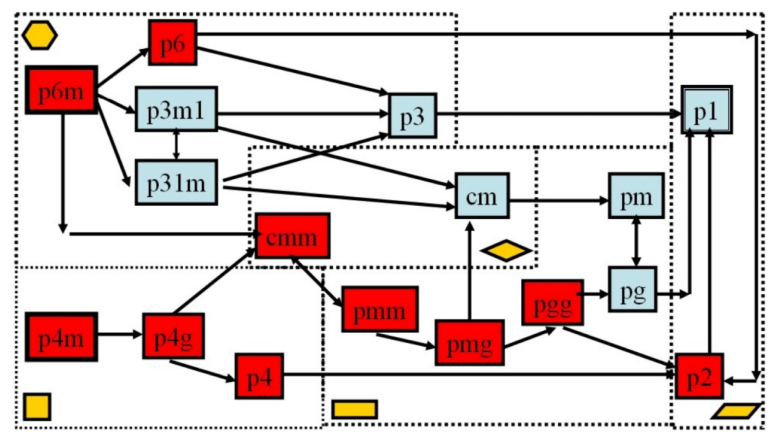

Figure 6: The subgroup relation graph. If an arrow points from any given box, $A$, toward any given box $B$, that means that $B$ 's symmetries are subset of $A$ 's symmetries.

1. Can people naively distinguish among the wallpaper groups? In other words, without using any knowledge of symmetry, are they still able to see the differences? If the answer is yes, then we can expect that, at the very least, more features than reflection play a role in symmetry perception. Further, if people are very good at telling apart the wallpaper groups, then it is possible that group-theoretic symmetry actually plays a role in perception. This integrates well with a computational cognition paradigm.

2. What features of symmetry drive the perception of symmetry? The presumption of previous studies is that reflection is the most important in perception, while Clarke's study claimed it was rotation. However, to the best of our knowledge, this is the first study to systematically isolate various symmetries. We will additionally investigate the role of the tile shape. 

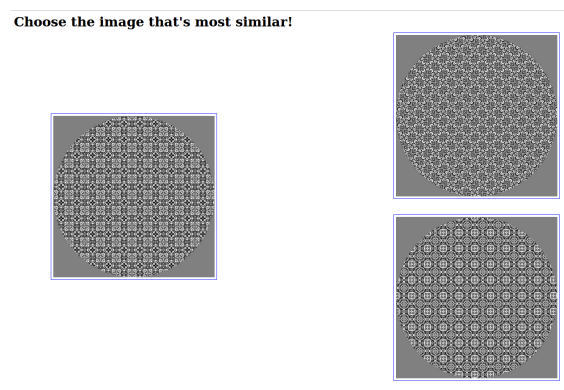

Figure 7: A screenshot of the experimental task. On the left is the target image. On the right on top is the distracter image, which is of a different group than the target. On the right on bottom is the goal image, which has the same group as the target.

\section{Experiments}

We designed an experiment where people had to distinguish between wallpaper groups. The goal of the task is to see which groups subjects can easily distinguish among and which groups are more challenging. In every trial, a subject was presented with two images from the same group and a third from one of the other sixteen groups. On the left, we have the target image, which is the image with which they compare the other two images to. On the right, there is the goal image, which always has the exact same group as the target image. Also, on the right is the distracter image, which has any other wallpaper group. Figure 7 shows what a single trial looks like.

We recruited 106 participants on Mechanical Turk to participate in the experiment for compensation. Each subject performed 272 trials. This allowed each trial to have every group as the distracter image for every other group. This allows us to determine which features of the groups cause difficulty.

To generate these 272 tasks, we created every possible combination task from a small set of images from each wallpaper group. Then, each participant who accepted the HIT on Mechanical Turk was assigned a set of tasks randomly, though they received exactly one task that had any given group for the goal and the distracter.

We introduced a time limit of five seconds to ensure that the subjects were performing the task intuitively without relying on formal knowledge. This should give participants plenty of time to click the one they felt was more similar. However, when the task was given to people who have been trained in distinguishing wallpaper groups, they reported that the time limit felt too short to determine which group was which. Thus, it is likely that the results are a result of perception rather than education.

As the task can be tiring, subjects were allowed one break halfway through the experiment. Each subject was paid $\$ 2$ and a bonus for each task they successfully completed beyond chance, to encourage people to try as hard as they could on the task, instead of simply guessing. They could earn up to $\$ 2$ by answering $100 \%$ correctly.

Unfortunately, this incentive did not work on all participants. We excluded 10 participants from the analysis due to behavior that seemed consistent with either a lack of effort or following the directions poorly. We used exclusion heuristics that identified participants that primarily clicked on either the top or bottom image, or if participants let a large portion of their tasks time out.

\section{Analysis}

We addressed our two research questions separately.

\section{Question 1}

Technically, the data we collected were asymmetric; that is, we have separate data from when group $A$ serves as the target and group $B$ serves as the distracter than when group $A$ serves as the distracter and group $B$ serves as the target. While we suspected this would be a very comparable task, we performed McNemar's test to determine if our data was symmetric. As it failed standard tests of significance $p>0.1$, we decided to pool the data. See Figure 9 for the non-symmetric accuracies.

To determine whether people could naively distinguish among wallpaper groups, we performed binomial t-tests comparing participants' actual performance to chance $(\pi=0.5)$. Using this paradigm, we found that $135 / 136$ tests were significant at the $p<0.05$ level; the last test was still significant at the $p<0.10$ level. We computed t-tests individually to check whether every group was distinguishable from every other, rather than if the average two groups were distinguishable. There was clear variation in these probabilities, although people were able to distinguish among the wallpaper groups significantly better than chance.

The most difficult pair for participants to distinguish between were P4M and PMM (see Figure 8 for an example). Interestingly, an expert given a small amount of time should have almost no trouble distinguishing them, as the lack of 4fold rotation is quite obvious in PMM. Across the 96 participants, only $56.8 \%$ of trials were successful. For comparison, the average accuracy among all trials was $76.8 \%$. However, there remains a possibility that certain groups are nearly indistinguishable to non-experts.

\section{Question 2}

To answer our second question, we used a Linear Mixed Effects Regression (GLMM) model. We tried to determine which symmetries contributed to accuracy. Thus, we used a comparison of each task's symmetries as the model.

1. 2-Fold Rotation, 3-Fold Rotation, 4-Fold Rotation, 6-Fold Rotation (True, False). In this case, groups with 6-Fold are guaranteed to have 3-Fold and 2-Fold.

2. Reflection, Glide Reflection, or nothing along the four main axes $\left(T_{1}, T_{2}, D_{1}, D_{2}\right)$ 


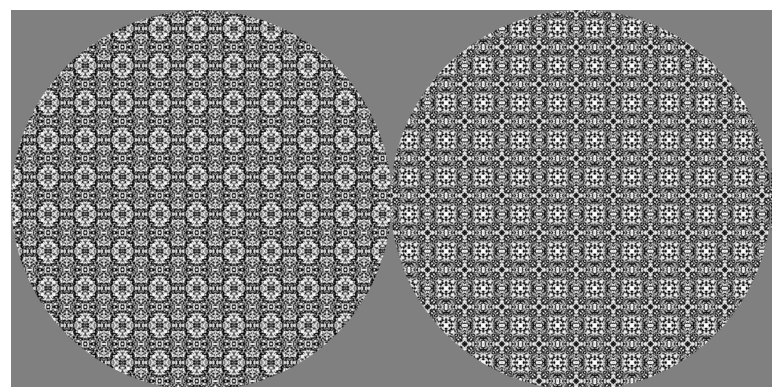

Figure 8: PMM on the left (note the tiles lack reflection on the diagonal axis), $\mathrm{P} 4 \mathrm{M}$ on the right.

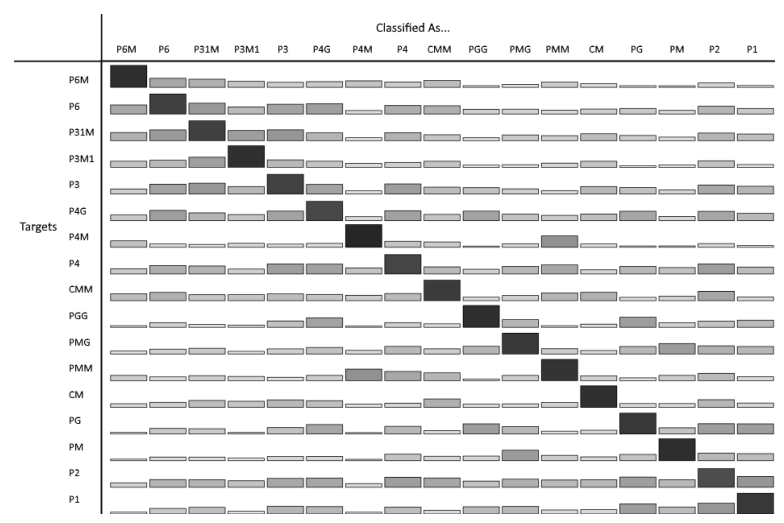

Figure 9: On the vertical we have the targets. On the top are the distracters. The bars represent the percentage of errors. The main diagonal, where the target and distracter are the same, represents the aggregate accuracy in labeling that group correctly. The darkness of the bar also corresponds to its value (higher is darker).

3. Tile Shape. This feature is not included in the grouptheoretic analysis, but might play a role

4. Subgroup distance, computed with Djikstra's algorithm using the subgroup relation graph found in Figure 1.

We included two random effect intercepts grouped by participant and the specific task (combined by-item and bysubject analysis).

The best model was identified according to Aikake Information Criterion (AIC), among the models including the pool of features described earlier. This best model included distance, but additionally included the $T_{1}$ axis, which would correspond to bilateral reflection symmetry, the $D_{1}$ axis, which is the positive diagonal, 4-fold rotation, and 3-fold rotation. See Table 2 for the fixed effects in a model including all possible features.

\section{Discussion}

There are several results from our study that require some explanation to integrate with the literature. Clarke et al. (2011) found that people have fairly high inaccuracy in matching the

\begin{tabular}{|l|rrrl|}
\hline & Estimate & Std. Error & $\mathrm{z}$ value & $\operatorname{Pr}(<|z|)$ \\
\hline (Intercept) & 0.95971 & 0.09950 & 9.645 & $<2^{-16 * * *}$ \\
\hline T1 & -0.24795 & 0.04150 & -5.975 & $2.30^{-9 * * *}$ \\
\hline T2 & -0.07746 & 0.04026 & -1.924 & 0.0544 \\
\hline D1 & -0.31416 & 0.04063 & -7.732 & $1.06^{-14 * * *}$ \\
\hline D2 & 0.10197 & 0.04431 & 2.301 & $0.0214^{*}$ \\
\hline 2fold & 0.01214 & 0.03540 & 0.343 & 0.7316 \\
\hline 3fold & -0.18056 & 0.04197 & -4.302 & $1.69^{-5 * * *}$ \\
\hline 4fold & 0.32353 & 0.04282 & 7.555 & $4.19^{-14 * * *}$ \\
\hline 6fold & -0.07538 & 0.04484 & -1.711 & 0.0870 \\
\hline tile & -0.07891 & 0.05556 & -1.420 & 0.1555 \\
\hline distance & 0.22420 & 0.01872 & 11.978 & $<2^{-16 * * *}$ \\
\hline
\end{tabular}

Table 2: Logistic Linear Mixed-Effects model predicting accuracy with by-item and by-subject random intercepts. (* implies $p<0.05, * *$ implies $p<0.01, * * *$ implies $p<0.001$ )

wallpaper groups together and that they seem to primarily rely on rotation in their strategy. However, Clarke's study relies on grouping images together. When presented with this task, it seems unlikely that most people would immediately choose to use seventeen groups. Indeed, the number of sets participants constructed varied wildly in Clarke's study, from 2 to 23 (Clarke et al., 2011). This makes it somewhat difficult to analyze the actual strategy participants used. Further, if wallpaper images are to be sorted into a different number of groups, various strategies might make sense. For instance, rotation may be a logical method with six groups, as there are six types of rotation a wallpaper can have. Importantly, Clarke's allows participants an unlimited amount of time, so that they can employ analytical reasoning. Therefore, perception is not successfully isolated. Lastly, due to working memory constraints, it would be difficult for participants to recall precisely what type of image corresponded to each group as they continued to sort. These confounds could explain why their participants seemed to have significantly higher error rates than ours.

In general, this paper advocates for a mathematically driven model of symmetry/pattern detection. We believe the subgroup distance to be one method to capture how humans perceive regularities. Importantly, it is not the only method and it may not even be the best one. In computing our subgroup distance, we made every edge in the hierarchy to be of equal distance, and then we used Dijkstra's algorithm. However, that's not necessarily the only way. While P1 and P2 differ by exactly one symmetry (the 2-fold rotation), P2 and PGG differ by three symmetries. Another possible view is to calculate the distance using the Hamming distance between each pair, rather than using the relation graph. Thus, there are multiple ways to operationalize the same idea, which complicates our explanation.

The model containing only subgroup distance outperformed the other simple models, so we find it likely that subgroup distance is the single most useful feature. However, it 
should be clear from our results that distance alone does not tell the whole story. Thus, we interpret the additional features in the converged model as having an important role in the process of pattern recognition compared to other symmetries. Human perception might use some quick heuristics involving these symmetries instead of doing a full analysis.

$T_{1}$ and $D_{1}$ are significant predictors (which correspond to the vertical and positive diagonal axes) while $T_{2}$ and $D_{2}$ are not (which correspond to the the horizontal and negative diagonal axes)? This could be explained by perception: perhaps symmetries along these axes are more noticeable to humans. However, it is important to note that there are substantial correlations among the features. In some cases, this should be obvious: if a wallpaper group has 6-fold rotation, it is guaranteed to have both 2 -fold rotation and 3 -fold rotation by definition. In the case of the reflection axes, there are substantial correlations (about 0.5 ) between $D_{1}$ and $D_{2}$ and between $T_{1}$ and $T_{2}$. Thus, while it's somewhat difficult to disentangle their independent effects, it seems clear that both $T_{1}$ and $D_{1}$ are at least more important, as the models including them instead of $T_{2}$ and $D_{2}$ have a lower AIC. However, $D_{2}$ is also a significant predictor even considering $D_{1}$, while $T_{2}$ is not a significant predictor while considering $T_{1}$. This could go back to faces: the diagonal axes $D_{1}$ and $D_{2}$ could represent faces at an angle. As mentioned earlier, there are biological reasons for believing humans are adept at recognizing faces (Kanwisher et al., 1997).

In the case of rotation, the most significant features were 3-fold and 4-fold. Interestingly, the effect of 4-fold rotation is positive (if both groups both have 4-fold rotation or both don't, accuracy improves), while the effect of 3-fold rotation is negative. This means that groups with 4-fold rotation are not necessarily easy to determine from groups without it, but they are easy to tell apart internally. This is very possibly due to 4-fold rotation's correlation with the other features.

For 3-fold rotation, it is possible that it plays a more significant role due to its lack of ubiquity. Many objects humans interact with in the modern world contain 4-fold rotation, to the point where perhaps it is so common that it is no longer a useful heuristic. On the other hand, 3-fold rotation is rarer, and thus may have some special significance.

Lastly, distance was highly significant in all models. This could suggest that people perceive these patterns' similarity based on their relationships rather than any individual features. Computer vision has long been inspired by wallpaper groups (Liu, Collins, \& Tsin, 2004; Schindler, Krishnamurthy, Lublinerman, Liu, \& Dellaert, 2008), and this result lends further credence to the idea that they play some fundamental role in human perception.

\section{Conclusion}

This paper argues that human pattern perception is sensitive to more types of symmetry than previously thought. To date, data on types of symmetry other than bilateral reflection were limited; however, we present evidence that humans can and do perceive the other types of symmetry. We found that all but one pair of wallpaper groups are reliably distinguishable $(p<0.05)$ and all are likely distinguishable $(p<0.1)$. After comparing models, we found that certain axes in reflection and certain types of rotation both play a role in symmetry detection. Notably, we also found that subgroup distance is a viable predictor of the accuracy of similarity judgments.

\section{Acknowledgements}

We acknowledge support from an National Science Foundation (NSF) Creative Research Award for Transformative Interdisciplinary Ventures (CREATIV), INSPIRE: Symmetry Group-based Regularity Perception in Human and Computer Vision (NSF IIS-1248076). We would like to thank Christopher Funk, Alexander G. Ororbia II, and Bill McDowell for their comments on an earlier draft.

\section{References}

Clarke, A. D., Green, P. R., Halley, F., \& Chantler, M. J. (2011). Similar symmetries: the role of wallpaper groups in perceptual texture similarity. Symmetry, 3(2), 246-264.

Federov, E. (1891). Symmetry in the plane. Proceedings of the Imperial St. Petersburg Mineralogical Society, 28(2), 245-291.

Ferguson, R. W., Aminoff, A., \& Gentner, D. (1996). Modeling qualitative differences in symmetry judgments. In Proceedings of the eighteenth annual conference of the cognitive science society (p. 12).

Kanwisher, N., McDermott, J., \& Chun, M. M. (1997). The fusiform face area: a module in human extrastriate cortex specialized for face perception. The Journal of Neuroscience, 17(11), 43024311.

Liu, Y., Collins, R. T., \& Tsin, Y. (2004). A computational model for periodic pattern perception based on frieze and wallpaper groups. Pattern Analysis and Machine Intelligence, IEEE Transactions on, 26(3), 354-371.

Liu, Y., Hel-Or, H., Kaplan, C. S., Van Gool, L., et al. (2010).

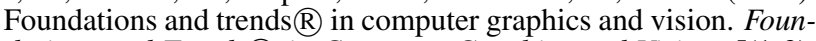
dations and Trends $\AA$ in Computer Graphics and Vision, 5(1-2), $1-195$.

Liu, Y., Lin, W.-C., \& Hays, J. (2004). Near-regular texture analysis and manipulation. In ACM Transactions on Graphics (TOG) (Vol. 23, pp. 368-376). ACM.

Morales, D., \& Pashler, H. (1999). No role for colour in symmetry perception. Nature, 399(6732), 115-116.

Schindler, G., Krishnamurthy, P., Lublinerman, R., Liu, Y., \& Dellaert, F. (2008). Detecting and matching repeated patterns for automatic geo-tagging in urban environments. In Ieee conference on computer vision and pattern recognition (pp. 1-7).

Treder, M. S. (2010, July). Behind the Looking-Glass: A Review on Human Symmetry Perception. Symmetry, 2(3), 1510-1543.

Wilson, H. R., \& Wilkinson, F. (2002). Symmetry perception: A novel approach for biological shapes. Vision research, 42(5), 589597. 